\title{
Quantum Gravity Equation In Schroedinger Form In Minisuperspace Description
}

\author{
S.Biswas ${ }^{* a), b)}$, A.Shaw ${ }^{* * a)}$, B.Modak $^{a)}$ and D.Biswas ${ }^{a)}$ \\ a) Department of Physics, University of Kalyani, West Bengal, India, Pin.- 741235 \\ b) IUCAA, Post bag 4, Ganeshkhind, Pune 411 007, India \\ * email: sbiswas@klyuniv.ernet.in \\ ** email:amita@klyuniv.ernet.in \\ (today)
}

Keywords : Quantum Cosmology; Quantum Gravity; Time; Minisuperspace; Wavefunction of the Universe

PACS No. - 04.60, 98.80 Hw

We start from classical Hamiltonian constraint of general relativity to obtain the EinsteinHamiltonian-Jacobi equation. We obtain a time parameter prescription demanding that geometry itself determines the time, not the matter field, such that the time so defined being equivalent to the time that enters into the Schroedinger equation. Without any reference to the Wheeler-DeWitt equation and without invoking the expansion of exponent in WKB wavefunction in powers of Planck mass, we obtain an equation for quantum gravity in Schroedinger form containing time. We restrict ourselves to a minisuperspace description. Unlike matter field equation our equation is equivalent to the Wheeler-DeWitt equation in the sense that our solutions reproduce also the wavefunction of the Wheeler-DeWitt equation provided one evaluates the normalization constant according to the wormhole dominance proposal recently proposed by us.

\section{INTRODUCTION}

There have been various attempts towards a resolution of the problem of time in quantum general relativity [1] 9 . The classical Hamiltonian constraint, in quantum theory of gravity, leads in an appropriate operator version to the Wheeler-DeWitt equation

$$
\hat{H} \Psi=0,
$$

where $\Psi$ is the wavefunction of the universe. The equation (1) when compared to a Schroedinger equation does not show the presence of time variable. In short, this is the problem of time in quantum gravity. Secondly, the interpretation of conservation of probability remain obscure without external time. Towards a solution of the problem of time in quantum gravity, a time variable $t$ is obtained [10,11 by performing an appropriate canonical transformation in which conjugate momentum $p_{t}$ occurs linearly such that

$$
H=H_{r}+p_{t}=0
$$

in which $H_{r}$ is the reduced Hamiltonian with conjugate momentum occurring quadratically. Upon quantization this becomes

$$
\hat{H} \Psi=o \Leftrightarrow\left(\hat{H}_{r}+\frac{\hbar}{i} \frac{\partial}{\partial t}\right) \Psi=0 .
$$

Though this approach has been successful in cylindrical gravitational waves or eternal blackholes, its general viability remains unclear. In the second approach one starts with the Wheeler-DeWitt equations and looks at a sensible concept of time. In this approach the normalization of the wavefunction which in turn requires an interpretation of 'probabilities' still remains unclear. Recent trend suggests to consider a solution of (3) in the form [12]

$$
\Psi(G, \phi) \simeq C(G) \exp \left[\left(\frac{i}{\hbar}\right) S_{o}(G)\right] \psi(G, \phi)
$$

where $G$ denotes the gravitational fields, $\phi$ stands for nongravitational fields and $C$ is a slowing varying prefactor. One then obtains with the identification

$$
i \hbar \nabla S_{o} \nabla \psi \equiv i \hbar \frac{\partial \psi}{\partial t} \simeq H_{m} \psi
$$


In deriving (5), one uses Wheeler-DeWitt equation with the WKB ansatz (4), but this has some limitations. The Wheeler-DeWitt equation (3) is linear in $\psi$ and hence if $\left|\psi_{1}\right\rangle$ and $\left|\psi_{2}\right\rangle$ are solutions of (3), then the superposition principle demands $|\psi\rangle=a\left|\psi_{1}>+b\right| \psi_{2}>$ to be also a solution. There is no a priori reason why the universe could not be in this state. But experience dictates that the universe behaves almost classically as far as macroscopic observations are concerned. For such a superposed states e.g., like (4), the derivation of (5) cannot be carried out. This is an inherent difficulty if one starts with the Wheeler-DeWitt equation. In the present paper we try to resolve the problem of time using a semiclassical point of view, avoiding the steps (1), (4) and (5) but keeping the fruits that these equations convey.

In section II we review critically the problem of time and state clearly the approach that we follow in the present paper. A good non-technical review [13 as well as a technical review 14 may be helpful to understand the many points followed in the present work. In section III we consider a model and solve the classical Hamiltonian constraint to obtain the prescription of time. This section deals with the emergence of complex paths for a model with gravity plus a minimally coupled scalar field in a FRW universe. In section IV we obtain the Schroedinger-Wheeler-DeWitt (SWD) equation for the model described in III. In section V we obtain the solution of the SWD equation including the basic aspect of the wormhole dominance proposal [15]. Assuming a Gaussian ansatz for SWD solution in the Lorentzian region, we show that it gives back the Hartle-Hawking wavefunction when continued to Euclidean spacetime. In section VI we make a concluding discussion.

\section{TIME IN QUANTUM GRAVITY}

The approach that we follow in the present work is termed as the emergence of time before quantization, though less persued in the literature. We therefore spend some words following the ref. 13,14. In quantum gravity dealing with the wavefunction of the universe, the traditional Copenhagen interpretation requires an 'observer' to carry out measurement; unfortunately we do not have this observer in quantum cosmology. A refinement replaces the 'observer' by a 'classical background' external to the system. This 'precondition of unambiguous communication', in Bohr's words, is not a well-placed argument.

Let us turn to the Everettian idea. According to this idea the particular classical realm, we observe or we live in, is basically just 'one component' of the universal state vector which always evolves deterministically, never collapsing. The criticism against the "preferred basis", chosen to serve as approximate position eigenstates of measurement, is now circumvented through the concept of decoherence. The decoherence allows a dynamically motivated specification of the 'preferred basis'. However, there are some subtle aspects: avoidance of the traditional no-hidden-variable theorems i.e., algebraic theorems in the tradition of Von Neumann, and the non-locality theorems in the tradition of Bell that must be avoided in the Everettian 'decoherence' mechanism. The decoherence is basically a diffusion of coherence (characteristic of quantum superposition principle) from a system to its environment. In the dancing coherent-ground of the 'system' plus the 'environment', macro-objects' initial superposition diffuses to the environment such that a 'variable' (position in ordinary quantum theory, scale factor in gravity) emerges nearest in sense to the classical counterpart. The above discussion refers to any system that deals with quantum to classical transition. For systems whose dynamics are described by any of the three fundamental interactions (electromagnetic, weak and strong) other than gravity, we have a time parameter with three characteristics. In the above three interactions the 'time' i.e., the time of everyday reality is considered (i) classical, (ii) non-dynamical and (iii) being the same in all models. But in quantum gravity the situation is rather obscure. We have here two broad problems: problem of time and the emergence of time. In classical general relativity the condition (i) is satisfied, whereas the conditions (ii) and (iii) are not so because the time enters into the Einstein equations dynamically just like other three position co-ordinates and different geometries (we understand here as different models) evolve with different facets of time. Thus for a given manifold with a spacetime structure we observe time as if having many fingures - called 'many fingured' time. This is briefly the problem of time. The problem with the 'emergence of time' is to embed/graft/bury an approximate physical time in timeless formulation of quantum gravity. By 'approximate' we intend to satisfy the conditions (i), (ii) and (iii), at least approximately.

At present we have two ways of embedding the time in quantum gravity formulation and it is carried out through canonical quantization. The two ways of embedding the time are to prescribe it 'before quantization' or to do the same 'after quantization'. Let us discuss the two procedures briefly. In quantum mechanics we follow the 'constrained quantization' in which the Hamilton of the system contains more variables than the physical degrees of freedom. For example, the classical general relativity constraint $H=0$ is converted to a constrained quantum equation by replacing $p_{i}=-i \frac{\partial}{\partial q_{i}}$. After that one introduces the time by a prescription. This is known as 'time after quantization'. Another attempt (of course less perused) is to solve the constraints before quantizing and to find the so called 'internal time' as a function of the canonical variables of general relativity such that this 'time' could serve as a time for the 
Schroedinger equation of quantized theory. This procedure is named as 'time before quantization'. We follow the second one in the present paper.

It is worthwhile to point out the reasons for choosing the embedding before quantization. In the approach 'before quantization', the constrained quantum equation is the Wheeler-DeWitt equation $\hat{H} \Psi=0$ and obviously this is timeless in character. We should have a way to interpret the wave function in a timeless way i.e., one has to settle between the 'Copenhagen interpretation' and the 'Everettian interpretation' that includes now the decoherence mechanism compared to the collapse of wavefunction of the former interpretation. This is the first problem. The second problems relates to the initial conditions for the Wheeler-DeWitt wavefunction, now classed by the words 'wavefunction debate' because of various boundary condition proposals; namely, Hartle-Hawking no boundary proposal [16], Vilenkin's tunneling proposal [17] and Linde's proposals [18]. Recently another proposal [15] has been made considering complex solutions of Hamilton-Jacobi equation of classical general relativity. In the proposal, termed as 'wormhole dominance proposal' attempts have been made to include complex solutions in the framework of the Wheeler-DeWitt equation with a general prescription of retaining 'allowable' and/or 'good' complex WKB paths. The 'wormhole dominance proposal' serves as a naive attempt. There were many attempts [19 21] to consider complex solution while evaluating the wave function of the universe using the path integral formulation but the recent attempts 222 suit nicely to the wormhole dominance proposal. The choosing of the paths in WKB formulation or in the HartleHawking path integral formulation is another problem and needs to incorporate complex solutions/complex paths. This is the second problem. In the present work we do not delve into the controversy while keeping only a salient feature in evaluating the normalization constant of Schroedinger-Wheeler -DeWitt solution. The third problem is related to the association of the wavefunction to the Lorentzian condition in quantum gravity.

In the past the approach to quantum cosmology considered the behaviour of the wavefunction, as a function of the overall scalefactor, $a$ of the 3 -metric $h_{i j}$, on the spacelike surface $\sum$. If the dependence on $a$ is exponential, the wavefunction corresponds to the Euclidean spacetime, while an oscillatory dependence on $a$ is interpreted as corresponding to a Lorentzian spacetime. However this distinction between exponential and oscillatory is not precise, and does not identify, which part of the wavefunction describes which physical situation [23]. Technically it amounts to asking whether a given spacelike surface is a part of a Lorentzian spacetime or a Euclidean spacetime. Our approach is: avoid the Wheeler-DeWitt equation, solve the classical constraints equations with a prescription for embedding the time, put the constraint equation in a form relating $p_{t}$ and $p_{\phi}$ (identifying $p_{t}$ and $p_{\phi}$ from Hamilton-Jacobi equation), quantize the constraint equation (we call it Schroedinger-Wheeler- DeWitt equation), adopt a boundary condition for the wavefunction with a view to decoherence, continue the wavefunction to the Euclidean regime and see whether the boundary conditions of classical spacetime (i.e., a large region) is any how related to the all quantum region (i.e., where both gravity and matter field are quantized). This approach allows to avoid many of the problems that have already been mentioned above. This is the main content of the present paper.

\section{MODEL}

We start with an action

$$
\begin{aligned}
I & =\int d^{4} x \sqrt{-g}\left[\frac{-R}{16 \pi G}-\frac{1}{2 \pi^{2}}\left(\frac{1}{2} \phi,{ }_{\mu} \phi^{\mu}+V(\phi)\right)\right] \\
& -\frac{1}{8 \pi G} \int_{\sum} d^{3} x \sqrt{h} K
\end{aligned}
$$

in a FRW universe

$$
d s^{2}=-d t^{2}+a^{2}(t)\left[\frac{d r^{2}}{1-k r^{2}}+r^{2}\left(d \theta^{2}+\sin ^{2} \theta d \phi^{2}\right)\right] .
$$

The Hamiltonian constraint corresponding to (6) now reads

$$
H=-\frac{1}{2 M a} P_{a}^{2}+\frac{1}{2 a^{3}} P_{\phi}^{2}-\frac{M}{2} k a+a^{3} V(\phi)=0 .
$$

In equation (8), $M=\frac{3 \pi}{2 G}$, where $G$ is Newton's constant, $k=0, \pm 1$ for flat, closed and open models, and $P_{a}=$ $-M a \dot{a}, P_{\phi}=a^{3} \dot{\phi}$. Identifying $P_{i}=\frac{\partial S}{\partial q_{i}}, q_{i}=a, \phi$, the Einstein-Hamilton-Jacobi equation is

$$
-\frac{1}{2 M}\left(\frac{\partial S}{\partial a}\right)^{2}+\frac{1}{2 a^{2}}\left(\frac{\partial S}{\partial \phi}\right)^{2}-\frac{1}{2} M k a^{2}+a^{4} V(\phi)=0 .
$$


Now to obtain the prescription like (5), we define a time operator consistent with Liouville's equation as

$$
\begin{aligned}
\frac{\partial}{\partial t} & =\sum_{i}\left(\frac{\partial H}{\partial P_{i}} \frac{\partial}{\partial q_{i}}-\frac{\partial H}{\partial q_{i}} \frac{\partial}{\partial P_{i}}\right) \\
& =\frac{\partial H}{\partial P_{a}} \frac{\partial}{\partial a}+\frac{\partial H}{\partial P_{\phi}} \frac{\partial}{\partial \phi}-\frac{\partial H}{\partial a} \frac{\partial}{\partial P_{a}}-\frac{\partial H}{\partial \phi} \frac{\partial}{\partial P_{\phi}} .
\end{aligned}
$$

that would satisfy (8) or (9). From (10) we get

$$
\begin{aligned}
\frac{\partial a}{\partial t} & =\frac{\partial H}{\partial P_{a}}=-\frac{P_{a}}{M a} \\
\frac{\partial \phi}{\partial t} & =\frac{1}{a^{3}} P_{\phi} \\
\frac{\partial P_{a}}{\partial t} & =-\left[\frac{P_{a}^{2}}{2 M a^{2}}-\frac{3 P_{\phi}^{2}}{2 a^{4}}-\frac{M k}{2}+3 a^{2} V(\phi)\right] \\
\frac{\partial P_{\phi}}{\partial t} & =-a^{3} \frac{\partial V}{\partial \phi}
\end{aligned}
$$

The above four equations along with the Hamiltonian constraint (8) determine t and also the paths characterized by $a$. Our aim is to find the extrema of the action when $a, t$ and $\phi$ are complex i.e., we are looking at complex four-metrics and complex fields on a real four dimensional manifold characterized by the coordinates $t, r, \theta, \phi$. To fix the origin of time defined above and to impose regularity condition on the four geometry at $a=0$, we choose the boundary condition as 22

$$
a(t=0)=0, \quad \frac{\partial a}{\partial t}=\beta i \text { at } t=0
$$

where $\beta= \pm 1$. For analytical simplicity we also assume that $V(\phi)$ is approximately constant, near about the region where (15) is satisfied. We find using (12) and (14)

$$
a^{3} \frac{\partial \phi}{\partial t}=\text { constant }
$$

If this constant is non-zero the boundary condition at $t=0$ leads $\frac{\partial \phi}{\partial t} \rightarrow \infty$. Hence we should have $\phi=$ constant. This implies $P_{\phi}=0$. Under this condition, when $V(\phi) \simeq V_{0}$ the solutions of (11)- (14) are called the zeroth order solutions. We write

$$
P_{a} \approx P_{a}^{0}=\frac{\partial S_{0}}{\partial a}
$$

where $S_{0}=S\left(a, V_{0}\right)$ is now identified as zeroth order action.

Restricting ourselves to $\mathrm{k}=+1$ universe and using the (13) and the constraint equation (9) we get

$$
a^{\prime 2}+a a^{\prime \prime}=4 a^{2} \frac{V_{0}}{M}-1
$$

where the prime now denotes differentiation with respect to $t$. Denoting its solution by $a_{0}(t)$ we get

$$
\begin{aligned}
a_{0}(t) & =\beta \sqrt{\frac{M}{2 V_{0}}} \sin \left(i \sqrt{\frac{2 V_{0}}{M}} t\right), \\
& =\frac{\beta}{\nu} \sin (i \nu t),
\end{aligned}
$$

where $\nu=\sqrt{2 V_{0} / M}$. This solution satisfies the boundary conditions

$$
a(0)=0, \quad a^{\prime}(0)=\beta i=\text { constant }, \quad a^{\prime \prime}(0)=0
$$

Let us construct the zeroth order action $S_{0}$ for the solution (19). Using (11) and (19) we find

$$
P_{a}^{0}=\frac{\partial S_{0}}{\partial a}=-M \beta a_{0}\left(2 a^{2} \frac{V_{0}}{M}-1\right)^{1 / 2} .
$$


We note that for $2 a^{2} \frac{V_{0}}{M}>1, P_{a}^{0}$ is real and is identified as classically allowed region. For $2 a^{2} \frac{V_{0}}{M}<1, P_{a}^{0}$ is imaginary and the corresponding region is identified as classically unallowed region. Thus we have the turning points at $a_{0}=0$ and at $a_{T}=\sqrt{\frac{M}{2 V_{0}}}$. We put the final boundary condition as $a_{0}=0$ at $t=0$, and $a_{0}=a_{f}$ at $t=t_{f}$. Integrating (21) we get

$$
S_{0}\left(a_{f}, V_{0}\right)=\frac{-M^{2} \beta}{6 V_{0}}\left(2 a^{2} \frac{V_{0}}{M}-1\right)^{3 / 2}+i \frac{M^{2}}{6 V_{0}}
$$

We have used $(-1)^{3 / 2}=\beta \quad i$. The two possible values of the action (due to the presence of $\beta$ ) have been the subject of immense controversy. However, we will not dwell upon this controversy. Recently there has also been some controversy with the 'factor ordering' problem [24] when one tries to construct the wavefunction from the WheelerDeWitt equation's solution with a given boundary condition proposal. The wormhole dominance proposal proposed by one of the authors 15] dwells upon complex path approach in the WKB approximation. The present work, we find, substantiate our previous work and confirm the prescription given in the wormhole dominance proposal. In the present work we discuss briefly the way how the complex paths enter into the description of wavefunction calculation of the Schroedinger-Wheeler-DeWitt equation. The details will be placed elsewhere. Here we mention the salient results that will be needed in the present discussion.

For $V(\phi)$ varying slowly such that $V(\phi)-V_{0}<<V_{0}$ we calculated the corrections $\delta S, \quad \delta^{2} S$ finding the first order correction to $a_{0}$ and $\phi_{0}$. From the calculation we observe that the semiclassical solution of Einstein equation allows complex solutions in both $a$ and $t$ space. The Hamilton-Jacobi function has not only real solutions but complex solutions that also contribute to the semiclassical path integral while evaluating the wavefunction of universe according to

$$
\Psi\left(G^{(3)}, \Phi\left(x \in G^{(3)}\right)\right)=\int e^{i S\left(G^{(4)}, \phi\left(x \in G^{(4)}\right)\right)} \delta \phi \delta G
$$

Here $G^{(4)}$ represents the four geometry and the fields $\phi$ defined on this four geometry are non-singular. There are some technical difficulties in choosing the complex paths in $a$-space in (23). However no unique prescription still has emerged. The question is to choose or find 'allowable' and 'good' paths, that contribute in (23). Finally, once one has calculated the wavefunction, it is still unclear how it resolves the problem of time, settles between the Copenhagen and Everettian approaches and interpret the timeless version of the Euclidean path integration. The wormhole dominance proposal provides a prescription for that in evaluating $\psi$ within the framework of complex semiclassical WKB approximation. A more technical discussion relating the complex paths and the evaluation of Euclidean path integral is reserved for concluding discussion in order to not track away from the main content of the paper. Let us try to understand the complex solutions considering (19) and (22). An important boundary condition is that both the initial and final value of $a$ must be real, keeping option open to include the complex paths in evaluating (23). Let us investigate the nature of the paths. We have $a\left(t_{i}=0\right)=0$ and take $a\left(t=t_{f}\right)=a_{f}$.

For a given $a_{f}=$ real, we find from (18)

$$
\begin{gathered}
\beta \cosh \left(\nu t_{R}\right) \sin \left(\nu t_{I}\right)=\nu a_{f} \\
\cos \left(\nu t_{I}\right)=0
\end{gathered}
$$

where $t_{f}=t_{R}+i t_{I}$. Corresponding to different $t_{i}$ given by (25) we have the same $t_{R}$ for a given real $a_{f}$. In otherwords, there are many different end points $t_{f}$ which give the same $a_{f}$ and many different contours to each of these end points. A numerical plot of the paths in the complex scale factor $(a)$ space of solution for various complex time $t$ will be found in [22]. In the cited work, they deal with complex solutions to study the classical evoluation without showing the construction of the wavefunction but in our work we construct the wavefunction taking the contribution of complex paths thereby exposing the connection between the small $a$ and large $a$ boundary conditions on the wavefunction.

Let us calculate the zeroth order action

$$
\begin{aligned}
S_{0}\left(a_{f}, \phi_{f}\right) & =\int_{0}^{t_{0}} P_{a}^{0} a_{0}^{\prime} d t \\
& =-M \int_{0}^{t_{0}} a_{0}\left(a_{0}^{\prime}\right)^{2} d t \\
& =-M \int_{0}^{a_{f}} a_{0} a_{0}^{\prime} d a_{0}
\end{aligned}
$$


and consider

$$
\Psi_{0}\left(a_{f}, \phi_{f}\right) \propto e^{i S_{0}}
$$

as the wavefunction of the universe (assuming that this is possible in the approach we follow). According to the wormhole dominance proposal, the wavefunction $\Psi$ given in (27) must be multiplied by those contributions of the contours in complex $a$-space (i.e., in complex $t$ space) which have the end points $a_{f}$ and initial point $a(0)=0$. Looking at (21), we find

$$
P_{a}^{0}=\frac{\partial S_{0}}{\partial a}=-M a_{0}\left(2 a_{0}^{2} \frac{V_{0}}{M}-1\right)^{1 / 2}
$$

which have turning points at $a_{0}=0$ and $a_{0}=\sqrt{\frac{M}{2 V_{0}}}=a_{T}$. Thus,

$$
S_{0}(\text { Complex paths })=S_{0}\left(a_{f}, 0\right)+S\left(a_{0}=0 \rightarrow a_{T} \rightarrow a_{0} \rightarrow a_{f}\right) .
$$

Here the first term is the contribution from the path that goes from $a=0$ to $a=a_{f}$ and the second term when evaluated corresponds to a loop path between $a=0$ and $a_{f}=\sqrt{\frac{2 V_{0}}{M}}$ times the first term in (29). When the repeated loops contribute we get a geometric series. Correspondingly the wavefunction is given by

$$
\Psi\left(a_{f}, \phi_{f}\right)=\frac{e^{i S_{0}\left(a_{T}, 0\right)}}{1-e^{2 i S_{0}\left(a_{T}, 0\right)}} \Psi_{0}
$$

considering repeated paths, to and fro, between $a=0$ and $a=a_{T}$. For the derivation of this result the reader is referred to [15]. The contribution $S_{0}\left(a_{T}, 0\right)$ gives the second term in $(21)$ and we observe that the imaginary part of the action determines the amplitude and the real part determines the phase of the wavefunction. The CWKB approach gives the extra term i.e., the denominator in (30). This term correctly takes into account the one loop quantum correction. Applying this technique to particle production in uniform electric field, we obtained [25] the Schwinger's one loop result [26] of $\operatorname{Im} L_{\text {eff }}^{(1)}$ term by term and observe that the particle production occurs due to the instability in the Hartle-Hawking vacuum. This lends support to keep the denominator in (30) which some authors (see concluding discussion) feel not necessary in the evaluation of the path integral.

To apply this approach in our case, we need a time contained description that will render a physical interpretation of $(1-\exp (2 i S))^{-1}$ term. Our approach is to treat the gravity as classical and retaining for $\phi$ the quantum behaviour and to obtain an quantum equation that contains the time.

\section{SCHROEDINGER-WHEELER-DEWITT EQUATION}

In our approach we keep only the $\delta S \equiv S_{1}$ term and consider the region in which $\nu a_{f} \gg 1$ and the gravity field could be treated classically such that $S_{1}<<S_{0}$ in the region of interest. By this we allow $a$ to be complex and is given by (19). We now eliminate $S_{1}$ through the following steps. In the region $\nu a_{f}>>1$ we take (see (11))

$$
\frac{\partial}{\partial t}=-\frac{1}{M a} \frac{\partial S_{o}}{\partial a} \frac{\partial}{\partial a} .
$$

At present we omit the subscript 0 in $a_{0}$ and will be introduced when required. Equation (31) now gives the prescription of time. The $\frac{\partial}{\partial t}$ is a directional derivative along each of the classical spacetimes which can be viewed as classical 'trajectories' in the gravitational configuration space. In view of (11)-(13), we identify the variable $t$ as the classical time parameter. Using (31), we now write

$$
\frac{\partial S}{\partial t}=-\frac{1}{M a} \frac{\partial S_{o}}{\partial a} \frac{\partial S}{\partial a}
$$

where $S$ is given in (9). Writing $S(a, \phi)=S_{o}(a)+S_{1}(a, \phi)$, we write (32) as

$$
\frac{\partial S}{\partial t}=-\frac{1}{M a}\left(\frac{\partial S}{\partial a}\right)^{2}+\frac{1}{M a} \frac{\partial S_{1}}{\partial a} \frac{\partial S}{\partial a} .
$$

We now substitute $\left(\frac{\partial S}{\partial a}\right)^{2}$ from (9) to get 


$$
\frac{\partial S}{\partial t}=-\frac{1}{a^{3}}\left(\frac{\partial S}{\partial \phi}\right)^{2}+M k a-2 a^{3} V(\phi)+\frac{1}{M a} \frac{\partial S_{1}}{\partial a} \frac{\partial S}{\partial a} .
$$

In the region of interest i.e., when $V(\phi) \approx V_{0}, P_{\phi}^{0}=0$, we write $P_{a} \approx P_{a}^{0}$ and using (13) we find

$$
\frac{1}{M a}\left(P_{a}^{0}\right)^{2}=2 a\left[\left(-P_{a}^{0}\right)^{\prime}+\frac{M k}{2}-3 a^{2} V_{0}\right] .
$$

Further neglecting $\left(\frac{\partial S_{1}}{\partial a}\right)^{2}$ term we get

$$
\begin{aligned}
\frac{1}{M a} \frac{\partial S_{1}}{\partial a} \frac{\partial S}{\partial a} & \simeq \frac{1}{M a} \frac{\partial S_{1}}{\partial a} \frac{\partial S_{0}}{\partial a} \\
& =\frac{1}{M a} \frac{\partial S_{0}}{\partial a} \frac{\partial S}{\partial a}-\frac{1}{M a}\left(\frac{\partial S_{0}}{\partial a}\right)^{2} \\
& =\frac{1}{M a} \frac{\partial S_{0}}{\partial a} \frac{\partial S}{\partial a}-\frac{1}{M a}\left(P_{a}^{0}\right)^{2}
\end{aligned}
$$

Using (32) to replace the first term in (36) by $-\frac{\partial S}{\partial t}$ and using (35) to replace the second term in (36) we get from (34)

$$
\frac{\partial S}{\partial t}=-\frac{1}{2 a^{3}}\left(\frac{\partial S}{\partial \phi}\right)^{2}-a^{3} V(\phi)+a\left(P_{a}^{0}\right)^{\prime}+3 a^{3} V_{0} .
$$

When $V(\phi) \approx V_{0}$, the Hamiltonian constraint is

$$
-\frac{\left(P_{a}^{0}\right)^{2}}{2 M a}-\frac{M k a}{2}+a^{3} V_{0}=0
$$

Using (38) in (35) we get

$$
\left(P_{a}^{0}\right)^{\prime}=M k-4 a^{2} V_{0}
$$

In the region, $4 a^{2} \frac{V_{0}}{M}>>1$, (we now consider $k=+1$ ) we have

$$
a\left(P_{a}^{0}\right)^{\prime}=-4 a^{3} V_{0}
$$

Putting this value in (37), we finally get

$$
\frac{\partial S}{\partial t}=-\frac{1}{2 a^{3}}\left(\frac{\partial S}{\partial \phi}\right)^{2}-a^{3}\left(V(\phi)+V_{0}\right)
$$

It is now straightforward to quantize (41) identifying $P_{t}=\frac{\partial S}{\partial t}$ and $P_{\phi}=\frac{\partial S}{\partial \phi}$. Upon quantization we get

$$
i \frac{\partial \Psi(a, \phi)}{\partial t}=\left[-\frac{1}{2 a^{3}} \frac{\partial^{2}}{\partial \phi^{2}}+a^{3}\left(V(\phi)+V_{0}\right)\right] \Psi(a, \phi) .
$$

In (42), $\Psi$ refers to the wavefunction of the universe, in which $a(t)$ is given by the solution of classical constraint equation. In this sense, apart from the extra term $V_{0}$ (that one does not have through 'time' after quantization) (42) takes the equivalent role as does the Wheeler-DeWitt equation. We will show this in the next section. In obtaining (42) we have nowhere used the Wheeler-DeWitt equation and the corresponding WKB ansatz for $\Psi$. Equation (42) is our Schroedinger-Wheeler-DeWitt equation. We need some boundary condition for the SWD wavefunction. We would start with a Gaussian ansatz and see what type of wavefunction does it lead to when continued to a region $2 a^{2} \frac{V_{0}}{M}<1$, where both gravity and the matter field are both quantum in nature. From the classical Einstein equation

$$
G_{\mu \nu}=R_{\mu \nu}-\frac{1}{2} g_{\mu \nu} R=k T_{\mu \nu}
$$

we observe that "geometry and matter" get coupled through (43). It is also a well known fact that the matter field is quantized and for that reason in equation (43) one writes $\left\langle T_{\mu \nu}\right\rangle$ on the right hand side and treats $g_{\mu \nu}$ as classical background. Keeping this in mind we argued that time is determined by the geometry itself. Let us be precise about the equation (42). The time that appears in (42) is the time that we use in quantum theory and is specified by the classical background $a(t)$ which in turn has been determined from the initial condition as if the many fingureness gets hidden in the $a(t)$ by the initial condition. The dynamicalness of $t$ has been transferred by demanding both $a(t)$ and $t$ complex and allowing $t$ to move both forward and backward direction. Whereas it is difficult to realize it in the framework of timeless Wheeler-DeWitt equation, but this interpretation works for equation (42). What is important to show that whether this time is buried in the Wheeler-DeWitt equation or not. We will take up this point in the next section. 


\section{SOLUTION OF SCHROEDINGER WHEELER-DEWITT EQUATION}

Let us now take the solution of (42)

$$
\Psi=N(t) e^{-\frac{\Omega(t)}{2} \phi^{2}}
$$

with a choice of $V(\phi)=\frac{\lambda}{2}\left(1-m^{2} \phi^{2}\right), \lambda$ being a constant. The time appearing in (42) is the physical time, at least in the region we are considering. Now substituting (44) in (42) we find

$$
\begin{gathered}
i \frac{d}{d t} \ln N=\frac{\Omega}{2 a^{3}}+a^{3} \lambda \\
i \frac{\partial \Omega}{\partial t}=\frac{\Omega^{2}+a^{6} \lambda m^{2}}{a^{3}} .
\end{gathered}
$$

Making an substitution

$$
\Omega=-i a^{3} \frac{\dot{y}}{y}
$$

and using the conformal coordinate $d t=a d \eta$, equation (46) gives

$$
y^{\prime \prime}+2 \frac{a^{\prime}}{a} y^{\prime}-\lambda m^{2} a^{2} y=0 .
$$

Taking an inflationary background $a(\eta)=-\frac{1}{\sqrt{\lambda} \eta}$, the solution $y$ is given by

$$
y=C_{1} \eta^{3 / 2 \pm \sqrt{9 / 4+m^{2}}} .
$$

For practical purposes $m^{2}<\frac{9}{4}$ and $\sqrt{9 / 4+m^{2}} \simeq\left(3 / 2+m^{2} / 3\right)$. Taking the negative sign in (49) and in conformal coordinate

$$
\Omega=-i a^{2}(\eta) \frac{y^{\prime}}{y}
$$

we get

$$
\Omega=-i m^{2} \sqrt{\lambda} \frac{a^{3}}{3} .
$$

Since $m^{2} \sqrt{\lambda}$ is small and $a$ is large and $\frac{d}{d t}=\sqrt{\lambda} a \frac{d}{d a}$, we get from (45)

$$
N=N_{o} \exp \left[-\frac{i a^{3} \sqrt{\lambda}}{3}\right]
$$

the integration constant $N_{o}$ has to be determined through a suitable initial condition. We now write down the solution of the SWD equation (42) as

$$
\begin{aligned}
\Psi & =N_{0} \exp \left[i \frac{m^{2} \sqrt{\lambda} a^{3}}{6} \phi^{2}-i \frac{a^{3} \sqrt{\lambda}}{3}\right] \\
& \simeq N_{0} \exp \left[-i \frac{a^{3}\left[\lambda\left(1-m^{2} \phi^{2}\right)\right]^{1 / 2}}{3}\right] \\
& \simeq N_{0} \exp \left[-i \frac{\left(a^{2} 2 V\right)^{3 / 2}}{3.2 V}\right] \\
& \simeq N_{0} \exp \left[-i \frac{\left(a^{2} 2 V-1\right)^{3 / 2}}{3.2 V}\right]
\end{aligned}
$$


In obtaining (53) we have made the approximation $\sqrt{\lambda}\left(1-\frac{1}{2} m^{2} \phi^{2}\right) \simeq\left(1-m^{2} \phi^{2}\right)^{\frac{1}{2}} \sqrt{\lambda}=\sqrt{2 V(\phi)}$ and $2 a^{2} V \simeq 2 a^{2} V-1$ for $2 a^{2} V>1$, which is quite valid in classically allowed region. This is what we meant earlier by saying 'the region of interest' while neglecting $S_{1}$ compared to $S_{o}$. Obviously the wavefunction (53) is not normalizable because of absence of real part of $\Omega$ in equation (51). The general procedure is to consider higher mode solutions of the scalar field. We take however the complex trajectory approach as in [15]. It should be pointed out that though we started with a Gaussian form, the wavefunction very closely resembles the WKB form that we guessed earlier (see (27)). To compare our result with that of the Wheeler-DeWitt equation, let us obtain the Wheeler-DeWitt equation corresponding to the above potential. We pass over a M-independent description with the following substitution in the Hamilton-Jacobi equation (9).

$$
S \rightarrow M S, \quad \phi \rightarrow M \phi^{\frac{1}{2}}, \quad V(\phi) \rightarrow M V(\phi) .
$$

The Wheeler-DeWitt equation that follows from (8) or (9) is

$$
\left[\frac{\partial^{2}}{\partial a^{2}}-\frac{1}{a^{2}} \frac{\partial^{2}}{\partial \phi^{2}}-a^{2}\left(1-a^{2} 2 V(\phi)\right)\right] \Psi=0 .
$$

As is evident from the classical equation (28) or the Wheeler-DeWitt equation (55), we find the two turning points at $a_{0}=0$ and at $a_{\mathrm{T}}=\frac{1}{\sqrt{2 V}}$. It should be pointed out that we have confined the discussions to regions in which the potential $V(\phi)$ can be approximated by a constant $V_{0}$, acting like a cosmological constant, so that the $\phi$ dependence in (55) can be effectively ignored. We now write (53) as

$$
\Psi=N_{0} e^{-i S_{e f f}}
$$

where

$$
S_{e f f}=\frac{\left(a^{2} 2 V-1\right)^{3 / 2}}{3 \times 2 V} .
$$

According to 'wormhole dominance' proposal, $N_{0}$ is given by

$$
N_{o}=\frac{\exp \left[-i S_{e f f}\left(a_{\mathrm{T}}, 0\right)\right]}{1-\exp \left[-2 i S_{e f f}\left(a_{\mathrm{T}}, 0\right)\right]}
$$

where

$$
S_{e f f}\left(a_{\mathrm{T}}, 0\right)=\left.S_{e f f}\right|_{0} ^{a_{\mathrm{T}}}
$$

Evaluating (59) we find

$$
N_{o}=\frac{\exp \left(\frac{1}{3 \times 2 V}\right)}{\left(1-e^{\frac{2}{3 \times 2 V}}\right)}
$$

taking $(-1)^{3 / 2}=-i$. Continuing in the region $2 a^{2} V<1$, we get

$$
\Psi=C_{1} e^{\frac{1}{3 \times 2 V}\left[1-\left(1-a^{2} 2 V\right)^{\frac{3}{2}}\right]},
$$

where

$$
C_{1}=\frac{1}{1-\exp \left(\frac{2}{3 \times 2 V}\right)}
$$

Equation (61) is the wavefunction of wormhole-dominance proposal. If we leave aside the $C_{1}$ term in (61), it gives the Hartle-Hawking wavefunction when $V(\phi)=$ Constant $=V$. In our earlier work 15 we have shown that the normalization constant $N_{o}$ thus obtained is consistent with Coleman and Klebanov's [27.28 arguments and we interpreted the constant $N_{o}$ as a manifestation of wormhole contribution to the wavefunction. The recent work [22] also supports our viewpoint.

Thus we have shown that the initial condition of SWD equation is fixed by the Gaussian anstaz 


$$
\Psi=N(t) e^{-\frac{\Omega}{2} \phi^{2}}
$$

in which a factor $N(t)$ is obtained taking the contributions of repeated reflections between the turning points. This contribution can be interpreted as due to wormhole contributions.

The superposition principle of quantum mechanics requires the existence of repeated reflections and here it is ensured

by the presence of turning points. The classical turning point $a=\frac{1}{\sqrt{2 V}}$ serves as a doorway to keep the universe's emergence in the classical regime. The quantum turning point $a=0$ acts as an entrance door of all quantum force supposed to arise from the other ensemble of universes.

An intuitive picture is that there is an ensemble of 'Quantum Universes' from which our universe tunnels retaining only the quantum property of uncertainty principle, the other quantum characteristics of ensemble of universe are thus still lying hidden and of course this applies to our universe also.

\section{DISCUSSION}

At present, all three standard proposals mentioned earlier are formulated in terms of a Euclidean path integral. This path integral depends crucially on how paths are chosen to evaluate the the integral. The Euclidean action for gravity is unbounded from below and hence a complex contour is generally necessary for convergence. No proposal does uniquely fix the contour for the evaluation of the path integral and as it stands does not define a unique wave function of the universe. Klebanov, Susskind and Banks [28] evaluated the path integral (in the $3+1$ case) considering multiple spheres configurations connected by the wormholes. However, Halliwell and Myers' [19] analysis reduces the path integral to a single path integration over the lapse function and the lapse has to be complex for convergence. Now as we find, the integration over the complex lapse equivalently be considered as an integration over the complex time (using some gauge condition for the lapse). The scale factor is then complex and a function of complex $t$. In the path integral formulation one evaluates the saddle points that represent the classical solutions. The result then depends on how the contour is chosen to include or exclude the saddles i.e., we require a suitable steepest-descent path. In 19] it is argued not to take the contributions of each saddles by summing them up as in [28].

In substance, while evaluating the wavefunction of the universe one must incorporate the complex solutions (i.e., complex $a(t)$ with complex $t$ ) remembering the fact that both the initial and final $a$ must be real. If we translate this viewpoint in terms of WKB formulation, this amounts to saying that the wavefunction at a real point $a$ is contributed not only by real WKB trajectories but also gets contribution from complex trajectories. Such a WKB formulation with complex trajectories was given in [29] with a heuristic expression for the solution of one dimensional Schroedinger equation. We used this technique in the wormhole dominance proposal 15] in a simple minisuperspace description. The technique of CWKB (complex path WKB approximation) applied to other fields (particle production in curved spacetime and in heavy ion scattering) gives remarkable results. It should be pointed out that the CWKB construction of the wavefunction of the universe is basically the same in spirit to that of [1.28], though more technical aspects remained to be investigated.

To elucidate the effectiveness of our proposal [15, we derive a Schroedinger type equation avoiding the WheelerDeWitt equation, the WKB ansatz and also the path integral formulation using the approach: time before quantization. With a Gaussian ansatz (dictated from decoherence) as a boundary condition, we obtained the normalization constant of our wavefunction using the wormhole dominance proposal. The resulting wavefunction seemingly resembles the Hartle-Hawking wavefunction of no boundary proposal. If we leave aside the $(1+\exp (2 i S))^{-1}$ term of $N_{0}$, the wave function results in the same situation as in [1]. (Halliwell and Myers worked in $2+1$ whereas we worked in $3+1$ dimensions) In our work multiple sphere configurations connected by wormholes are interpreted in terms of repeated reflections from the turning points. In the present work we are thus one step further to identifying the real and imaginary part of the action being associated with Euclidean and Lorentzian spacetime respectively. Though a naive attempt, we have been able to show that the time is buried in the structure of the Wheeler-DeWitt equation, and manifests itself through the Schroedinger equation dictated by the matter field Hamiltonian. The emergence of Hartle-Hawking wavefunction (better to say, a solution of the Wheeler-DeWitt equation) from the solution of the Schroedinger equation with a Gaussian ansatz is quite surprising and lends support to the Everettian idea of decoherence. The Copenhagen probabilistic interpretations also remain workable in our approach that had already been discussed in [15]. 
[1] J. J.Halliwell and S. W. Hawking, Phys. Rev. D31, 1777 (1985)

[2] C. Kiefer, Class. Quantum. Grav. 4, 1369 (1987)

[3] C. Kiefer, Phys. Rev. D46, 1658 (1992)

[4] C. Kiefer, Phys. Rev. D45, 2044 (1992)

[5] C. Kiefer, D. Polarski and A. A. Starobinsky, gr-qc/9802003 (1998)

[6] S. Wada, Nucl. Phys. B276, 729 (1986)

[7] T. P. Singh and T. Padmanabhan, Annals of Physics 196, 296-344 (1989) and references therein

[8] T. Padmanabhan, Phys. Rev. D39, 2924 (1989)

[9] T. Padmanabhan, Pramana - J. Phys. 35, L199 (1990)

[10] C. Kiefer,in 'Time, Temporality, Now' edited H Atmanspacher and E Ruhnau (Springer, Berlin), pp 227-240 (1997)

[11] K. V.Kuchar, in : Proceeding of the fourth Canadian Conference on General Relativity and Relativity Astrophysics, ed. by Kunstatter G Vincent D Williams J (World Scientific, Singapore), p 211-314 (1992)

[12] C. Kiefer, in Proceedings of the 10th seminar on Relativistic Astrophysics and Gravitation, Potsdam, 1991, edited by S.Gottloeber, J.P. Muecket and V. Mueller (World Scientific, Singapore, 1992)

[13] J. Butterfield and C.J. Isham, gr-qc/9901024 (1999)

[14] C.J. Isham, gr-qc/9210011 (1992)

[15] S. Biswas, B. Modak and D. Biswas, Phys. Rev. D55, 4673 (1996)

[16] J.B. Hartle and S.W. Hawking, Phys. Rev. D28, 2960 (1983)

[17] A. Vilenkin, Phys. Rev. D33, 3560 (1986); Phys. Rev. D37, 888 (1988)

[18] A.D. Linde, Sov. Phys. JETP 60,211 (1984); Lett. Nuovo Cimento 39, 401 (1984)

[19] J.J. Halliwell and R.C. Myers, Phys. Rev. D40, 4011 (1989)

[20] G.W. Lyons, Phys. Rev. D46, 1546 (1992)

[21] J.J. Halliwell and J. Louko, Phys. Rev. D42, 3997 (1990)

[22] W.G. Unruh and M. Jheeta, gr-qc/9812017 (1998)

[23] R.Bousso and S.W. Hawking, Phys. Rev. D59, 103501 (1999)

[24] N. Kontoleon and D.L. Wiltshire, Phys. Rev. D59, 063513 (1999)

[25] "Schwinger's result on particle production from complex paths WKB approximation" by S.Biswas, A.Shaw and B.Modak, submitted in Class. Quant. Gravity (1999)

[26] J. Schwinger, Phys. Rev. 82, 'On Gauge invariance and vacuum Polarization', 664 (1951)

[27] S. Coleman, Nucl. Phys. B310, 643 (1988)

[28] I. Klebanov, L. Susskind, and T. Banks, Nucl. Phys. B317, 665 (1989)

[29] J. Knoll and R. Schaeffer, Ann. Phys. (N.Y.) 97, 307 (1976)

\section{Acknowledgment}

A. Shaw acknowledges the financial support from ICSC World Laboratory, LAUSSANE during the course of the work. 